\section{Mycobacterial sensing}

Bacteria infecting the cytosol can be detected through their metabolic products in the form of cyclic dinucleotides such as c-di-AMP. In Nature Medicine, Bishai and colleagues find that mycobacteria also produce c-di-AMP, which is released into the cytosol of infected macrophages. By using mycobacteria either deficient in or overexpressing the gene responsible for c-di-AMP production, the authors observe that type I interferon (IFN-I) secretion by infected macrophages is correlated with the amount of c-di-AMP. Overexpressing bacteria show reduced virulence and growth in vivo, whereas the knockouts have increased virulence. Detection of c-di-AMP is via a helicase (DDX41)STING-mediated pathway but independent of the cytosolic pathogen sensor cGAS. Sensing of mycobacterial c-di-AMP results in the activation of autophagy. These data suggest that the sensing of c-di-AMP is also important for anti-mycobacterial responses and results in a potentially protective IFN-I and xenophagy response.

Nat. Med. (2 March 2015) doi:10.1038/nm.3813

\section{IL-33 supports $T_{H} 1$ cells}

The alarmin interleukin-33 (IL-33) is most typically associated with the initiation and enhancement of type II and T helper $2\left(\mathrm{~T}_{\mathrm{H}} 2\right)$ responses. In the Proceedings of the National Academy of Sciences, Löhning and colleagues find that IL-33 can also drive $\mathrm{T}_{\mathrm{H}} 1$ effector function. $\mathrm{T}_{\mathrm{H}} 1$ cells generated in a lymphocytic choriomeningitis virus (LCMV) model express a functional IL-33 receptor (ST2), albeit transiently and at lower amounts than that seen on $\mathrm{T}_{\mathrm{H}} 2$ cells. Expression of ST2 by $\mathrm{T}_{\mathrm{H}} 1$ cells is dependent on expression of the transcription factors STAT4 and T-bet. ST2 is not required for $\mathrm{T}_{\mathrm{H}} 1$ differentiation or homeostatic maintenance, but its absence impairs their effector function upon LCMV infection. Accordingly, LCMV-specific $\mathrm{T}_{\mathrm{H}} 1$ cells deficient in ST2 mediate less immunopathology after infection. Therefore, in addition to its wellestablished roles driving type II responses, IL-33 also seems in some other contexts to drive $\mathrm{T}_{\mathrm{H}} 1$ cells functions.

$Z F$

Proc. Natl. Acad. Sci. USA (17 March 2015) doi:10.1073/pnas.1418549112

\section{TREM2 function in microglia}

Myeloid cells, including microglia, express the surface receptor TREM2, a member of the immunoglobulin superfamily that signals via the adaptor DAP12 and phosphotyrosine kinase Syk. In Cell, Colonna and colleagues show that an Alzheimer's disease (AD)-associated variant (TREM2 R47H) exhibits defective recognition of anionic lipids, such as phosphatidylserine, sulfatides and sphingomyelin, that are exposed upon neuronal damage. Defective TREM2 activity results in poor microglial activation and compromises microglial survival when the availability of colony-stimulating factor 1 (CSF-1) is low, as during microgliosis. The consequence of such defective microglial activation is reduced clearance of $\beta$-amyloid plaques, which might explain the association with AD.

$L A D$

Cell 160, 1061-1071 (2015)

\section{Developmental stage matters}

Expression of the immune regulator Aire during the first few weeks of life is necessary and sufficient to prevent autoimmunity in Aire ${ }^{-l-}$ mice. In Science, Yang et al. show that perinatal Aire expression promotes the generation of regulatory $\mathrm{T}$ cells $\left(\mathrm{T}_{\text {reg }}\right)$ with a functional, transcriptome and activation profile distinct from that of $\mathrm{T}_{\text {reg }}$ cells produced in adult mice. Depletion of $\mathrm{T}_{\text {reg }}$ cells on days $0-10$ of life induces autoimmunity similar to that of Aire ${ }^{-/-}$mice, which is rescued only by perinatal wildtype $\mathrm{T}_{\text {reg }}$ cells and not by adult wild-type or perinatal Aire ${ }^{-/-} \mathrm{T}_{\text {reg }}$ cells. Perinatal $\mathrm{T}_{\text {reg }}$ cells are more proliferative, are more suppressive, have a higher expression of genes associated with $\mathrm{T}_{\text {reg }}$ effector function and persist in adult mice. The perinatal medullary thymic epithelial cells (mTECs) have a different, more diverse peptide repertoire compared to adult mTECs that is due to more effective loading into the MHC class II antigen-binding groove (owing to a lower ratio of the DO:DM molecular chaperones), resulting in a different TCR repertoire in the two age groups. Thus, perinatal $\mathrm{T}_{\text {reg }}$ cells have different repertoires, and may also guard distinct tissues or organs, compared to adult $\mathrm{T}_{\text {reg }}$ cells. Science (19 March 2015) doi:10.1126/science.aaa7017

\section{Necroptosis regulator}

Phosphorylation of the serine-threonine kinase RIP3 is required for the recruitment of the effector protein MIkI to the RIP1-RIP3 necrosome complex and necroptosis. In Nature Cell Biology, Han and colleagues show that the protein Ppmb1 functions as a phosphatase that restricts the autophosphorylation or tumor necrosis factor (TNF)-induced phosphorylation of RIP3 in the necrosome complex. Ppm 1b interacts with RIP3 and dephosphorylates it directly, but it does not bind to other proteins in the complex, such as RIP1, Fadd, caspase-8 and MIkl. Deletion of Ppm1b does not affect assembly of the necrosome, but it increases spontaneous and TNF-induced phosphorylation of RIP3 and recruitment of Mlkl to the necrosome in peritoneal and bone marrow-derived macrophages, mouse embryonic fibroblasts and L929 cells. Ppm1b-deficient mice are more susceptible to TNFinduced cell death, and this effect is completely prevented on a RIP3-deficient background. How Ppm1b activity is regulated during necroptosis remains unclear.

Nat. Cell Biol. (9 March 2015) doi:10.1038/ncb3120

\section{Targeting Nur77 in sepsis}

Mice lacking the nuclear receptor Nur77 are more susceptible to lipopolysaccharide (LPS) and systemic bacterial challenge. In Nature Chemical Biology, Li et al. reveal a negative feedback mechanism involving Nur77 interaction with the transcription factor NF-кB p65 subunit to interfere with its ability to bind to NF- $\kappa \mathrm{B}$ cis-regulatory sites and thereby limit expression of proinflammatory mediators. LPS-mediated activation of the MAP kinase p $38 \alpha$ leads to phosphorylation of Nur77 at threonines 27 and 143, impeding its ability to interact with p65. However, a synthetic molecule called PDNPA binds to Nur77 and acts as a competitive inhibitor preventing Nur77 interaction with p38 $\alpha$. PDNPA suppresses expression of proinflammatory cytokine genes in macrophages and reduces sepsis in mice. These findings suggest a means to prevent excessive cytokine production during episodes of acute inflammation.

$L A D$

Nat. Chem. Biol. (30 March 2015) doi:10.1038/nchembio.1788 